\title{
CALIDAD DE LOS DATOS DEL INSTITUTO NACIONAL DE ESTADÍSTICA PARA LA ELABORACIÓN DE LOS INDICADORES DE SALUD PERINATAL: PEQUEÑO Y GRANDE PARA SU EDAD GESTACIONAL (*)
}

\section{Sol Pía Juárez $(1,2)$}

(1) Centro de Estudios sobre Equidad en Salud (CHESS). Universidad de Estocolmo e Instituto Karolinska. Suecia.

(2) División de Medicina Ambiental y Ocupacional. Facultad de Medicina. Universidad de Lund. Suecia.

(*) Este trabajo se realizó con la financiación de la beca pre-doctoral I3P del Consejo Superior de Investigaciones Científicas y el apoyo del SIMSAM-Lund early-life.

La autora declara que no existen conflictos de interés

\section{RESUMEN}

Fundamentos: Los indicadores perinatales de peso relativo como pequeño y grande para la edad gestacional (PEG, GEG) son preferibles frente a medidas de peso absolutas (bajo peso, macrosomia). El objetivo de esta investigación fue evaluar si los datos del Instituto Nacional de Estadística (INE) tienen la fiabilidad suficiente para estimar indicadores de peso relativo y si la calidad de la información que comunican los progenitores varía según sus características sociodemográficas.

Métodos: Se comparó la información de 6.339 nacimientos del Hospita Clínico San Carlos de Madrid (2005-06) con el registro de nacimientos del INE (con un éxito del $95 \%$ ). Se evaluaron medidas de validación y acuerdo para los indicadores de bajo peso (BP $<2500$ gr), macrosomia $(>4.500$ gr), PEG (<percentil 10) y GEG (>percentil 90). Se realizó un análisis de regresión logísticas

Resultados: Las prevalencias estimadas con los datos del hospital fueron: BP $(6 \%)$, macrosomia $(0,5 \%)$ PEG $(1 \%)$ y GEG $(15 \%)$ y con datos del INE: $5 \% 0,5 \% 2 \% 12 \%$, respectivamente. La especificidad: $80 \%, 78 \%$, $24 \%, 82 \%$. El estadístico Kappa: BP (83\%), macrosomia (79\%), PEG $(24 \%)$ y GEG (82\%). La omisión e incorrecta declaración varió según la nacionalidad y la situación laboral de los padres (OR entre 1,5 y 2,2).

Conclusiones: El INE sobreestimaría la prevalencia de PEG e infraestimaría la de GEG. El acuerdo entre las fuentes es muy bueno para BP, macrosomia y GEG y moderadamente bueno para PEG. Tanto la omisión como los errores en la declaración del peso y la edad gestacional varían en función de las características sociodemográficas de los padres.

Palabras clave: Pequeño para su edad gestacional.Grande para su edad gestacional. Macrosomia. Bajo peso. Peso al nacer. Validación.

Correspondencia

Sol Pía Juárez

Sveaplan, Sveavägen 160, Floor 5

SE-106 91 Stockholm

Sweden

sol.juarez@chess.su.se

\section{ABSTRACT}

\section{Quality of the Spanish Vital Statistics to Estimate Perinatal Health Outcomes: Small and Large for Gestational Age}

Background: Relative measures of birthweight (small and large-forgestational age, SGA-LGA) are increasingly preferred to absolute measures (low birthweight, macrosomia). In this study we assess whether the national vital statistics provided by the Spanish National Statistical Institute (INE) reliably estimate SGA and LGA. Also, we will assess whether missing data (selection) and misreported information (bias) are systematically associated with parental socioeconomic information.

Methods: We linked the information on 6,339 births at the Hospital Clínico San Carlos of Madrid (2005-06) with the vital statistics records (successful for the $95 \%$ of the observation). Validity measures and concordance were estimated for low birthweight (LBW, $<2500$ gr), macrosomia $(>4500$ gr), SGA ( $<10$ th percentile) and LGA ( $>90$ percentile). Logistic regressions were fitted.

Results: The prevalence estimated with the hospital data were: LBW $(6 \%)$, macrosomia $(0.5 \%)$ SGA $(1 \%)$ and LGA (15\%) and, with the data from INE: $5 \% 0.5 \% 2 \% 12 \%$ respectively. Kappa statistics: LBW (83\%), macrosomia (79\%), PEG (24\%) and LGA (82\%). Missing and misreported data vary with parental nationality and their situation in the labor market (OR between 1.5 y 2.2).

Conclusions: Vital statistics overestimate the prevalence of SGA and underestimate the prevalence of LGA. The concordance between the sources is very good for low birthweight, macrosomia and LGA, and moderately good for SGA. Both missing and misreported birthweight and gestational age are associated with parental socioeconomic characteristics.

Keywords: Small-for-gestational age. Large-for-gestational age. Macrosomia. Low-birthweight. Birthweight. Gestational age. Validation. 


\section{INTRODUCCIÓN}

El peso al nacer es una de las variables más utilizadas para valorar la salud perinatal $^{1}$. Sin embargo, se la considera una medida incompleta como indicador de crecimiento fetal cuando no tiene en cuenta las semanas de gestación ${ }^{2,3}$. Pequeño (PEG) y grande para la edad gestacional (GEG) se consideran indicadores alternativos a bajo peso y macrosomia ${ }^{4-6}$. De hecho, en algunos contextos, como en Canadá, actualmente se excluye "bajo peso" de los informes de monitorización perinatal en favor de $\mathrm{PEG}^{3,6}$.

En el año 2012 se publicó un artículo en el que se formulaba una serie de advertencias en relación al uso del bajo peso al nacer $\mathrm{y}$ otros indicadores relacionados en investigación de salud ${ }^{3}$. Sin embargo, los autores no discutieron la importancia de la calidad de los datos disponibles en la elección del indicador de estudio (bajo peso vs PEG) $\mathrm{y}$, hasta la fecha, no hay trabajos que hayan evaluado específicamente este aspecto.

En España, la información sobre nacimientos publicada por el Instituto Nacional de Estadística (INE) procede de la información declarada por los padres en el Boletín Estadístico de Partos durante la declaración del nuevo nacimiento en el Registro Civil ${ }^{7}$. Este procedimiento pone en cuestión la fiabilidad de la información sobre el peso al nacer y la edad gestacional. Aunque una validación previa de esta fuente (con datos hospitalarios) mostraba una aceptable concordancia en los principales indicadores perinatales (bajo peso y pre-término) ${ }^{8}$, dicho estudio no consideró medidas relativas de peso (PEG y GEG).

Evaluar si los datos del INE afectan a los indicadores de peso relativo al nacer es crucial a la hora de decidir qué indicadores son los más apropiados para monitorizar la salud perinatal en España. Identificar si los errores y las omisiones tienen un carácter sistemático permite conocer sesgos evitables que afectan a la calidad del registro de nacimientos en materia de salud perinatal (y, por tanto, a su correcta monitorización) así como identificar posibles intervenciones para mejorar la calidad del registro.

El objetivo de esta investigación fue evaluar si los datos del Instituto Nacional de Estadística (INE) tienen la fiabilidad suficiente para elaborar indicadores de peso relativo y si la calidad de la información que comunican los progenitores varía según sus características sociodemográficas.

\section{MATERIAL Y MÉTODOS}

Mediante un acuerdo de colaboración entre el Hospital Clínico de Madrid, el Consejo Superior de Investigaciones Científicas y el Instituto de Estadística de la Comunidad de Madrid, se elaboró una base de datos. Se cruzó nominalmente la información de nacimientos procedente del hospital durante los años 2005 y 2006 con sus correspondientes registros de nacimientos registrados por el Instituto de Estadística de la Comunidad de Madrid (IEM). Se eliminaron los campos relativos a la información nominal de la madre para garantizar el secreto estadístico.

El IEM es encargado de reunir e informatizar los datos recogidos en el Boletín Estadístico de Partos que los progenitores rellenan en el Registro Civil. Una vez informatizados los datos, el IEM envía los ficheros al Instituto Nacional de Estadística que, posteriormente, elabora las estadísticas del Movimiento Natural de la Población $(\mathrm{MNP})^{9}$. Tanto la normalización como el enlace entre los ficheros del hospital y los del IEM se utilizó una herramienta desarrollada por el IEM (Padronco) ${ }^{10}$, la cual utiliza un método determinista con algoritmos que permiten diferentes grados de coincidencia. El enlace se realizó en el IEM por la autora de este trabajo. Del total de 6.339 nacimientos $5.991(95 \%)$ fueron exitosamente cruzados. El $80 \%$ de las observaciones tuvieron una coincidencia en los campos comunes entre $80-90 \%$ (nombre y apellidos de la 
madre y fecha de nacimiento). El 20\% restante tuvo unos niveles de coincidencia inferiores pero fueron supervisados manualmente. Los campos peso al nacer y edad gestacional no se utilizaron para vincular la información con el fin de evitar sesgos en los resultados ${ }^{11}$. La muestra final resultó ser representativa de los nacimientos ocurridos en el mismo período en el conjunto de la Comunidad de Madrid en cuanto a la edad de la madre y la nacionalidad y ocupación de los padres.

Se excluyeron las muertes fetales tardías $(\mathrm{n}=35)$ y los nacimientos múltiples $(n=202)$, ya que éstos últimos comparten la información de la madre, dificultando su correcta identificación. Se excluyeron de la fuente hospitalaria las observaciones cuya combinación de peso y edad gestacional resultaron biológicamente inverosímiles, así como las observaciones sin información sobre peso y edad gestacional $(n=113)$ y sexo del nacido $(n=2)$. Definimos como combinaciones biológicamente inverosímiles las de peso y edad gestacional que resultaron estar por encima o por debajo de los umbrales publicados en curvas de diversas poblaciones $^{12-15}$. Del total de 5.639 nacidos, con el próposito de evaluar PEG y GEG, se excluyeron observaciones correspondientes a las semanas 26 y 42 en la medida en que los estándares utilizados no tenían información para estas semanas. La muestra final fue de 5.626 nacimientos.

Se estimaron indicadores derivados del peso al nacer como bajo peso $(<2.500 \mathrm{gr})$, macrosomia ( $>4.500$ gr), pequeño para su edad gestacional (peso por debajo del percentil 10 en cada edad de gestación) y grande para su edad de gestación (por encima del percentil 90). Estas últimas fueron definidas de acuerdo con los estándares publicados para la población española específicas por $\mathrm{sexo}^{12}$. Ambas fuentes cuentan con la misma información: peso en gramos y semanas de gestación completas.

La calidad de la información proceden- te del INE se comparó con los datos del hospital mediante proporciones de bajo peso al nacer, macrosomia, PEG y GEG. Se evaluó su grado de acuerdo (mediante el estadístico Kappa) y se estimaron medidas de validez (sensibilidad, especificidad y valores predictivos positivos). Se ajustaron regresiones logísticas para estimar odd ratios (OR) e intervalos de confianza al 95\% (IC95\%). Las variables disponibles incluidas en los análisis fueron la nacionalidad, edad y situación laboral de los progenitores, estado civil, número de orden de nacimiento en el grupo familiar, sexo del recién nacido y el tiempo trascurridos desde el nacimiento hasta su declaración en el Registro Civil (en días).

\section{RESULTADOS}

En la tabla 1 se comparan las prevalencias de los indicadores de salud y las medidas de validez entre el hospital y el INE. Las prevalencias estimadas con los datos del hospital fueron, respectivamente, para bajo peso (6\%), macrosomia $(0,5 \%), \mathrm{PEG}$ (1\%) y GEG (15\%). Las correspondientes prevalencias estimadas con los datos del INE fueron para bajo peso (5\%), macrosomia $(0,5 \%)$, PEG (2\%) y GEG $(12 \%)$. Las diferencias entre las fuentes fueron estadísticamente significativas para PEG y GEG. El nivel de acuerdo general fue muy bueno para bajo peso al nacer (Kappa $=83 \%)$, bueno para macrosomia (79\%) y GEG $(75 \%)$, y moderadamente bueno para PEG (41\%). La especificidad fue notablemente baja para PEG (24\%) y alta para GEG $(82 \%)$, seguidos por bajo peso $(80 \%)$ y macrosomia $(78 \%)$. Los valores de sensibilidad fueron consistentemente altos $(>90 \%)$ en todos los indicadores. Los valores predictivos positivos fueron respectivamente para macrosomia $(99,8 \%)$, bajo peso $(98,7 \%)$, PEG (98,5\%) y GEG (97\%).

La tabla 2 muestra los resultados de las regresiones logísticas ajustadas para identificar las variables asociadas con los datos faltantes y con una incorrecta clasifica- 


\begin{tabular}{|c|c|c|c|c|c|c|c|c|}
\hline \multicolumn{9}{|c|}{$\begin{array}{r}\text { Proporciones y me } \\
\mathbf{y}\end{array}$} \\
\hline \multicolumn{5}{|c|}{ Proporciones } & \multirow{2}{*}{\multicolumn{4}{|c|}{ Medidas de validez ${ }^{\dagger}$}} \\
\hline \multirow[b]{2}{*}{ Peso al nacer } & \multicolumn{2}{|c|}{ Hospital } & \multicolumn{2}{|r|}{ INE } & & & & \\
\hline & $\mathrm{n}$ & $\begin{array}{c}\% \\
\text { IC- } 95 \%\end{array}$ & $\mathrm{n}$ & $\begin{array}{c}\% \\
\text { (IC-95\%) }\end{array}$ & Sensibilidad & Especificidad & VPP & Kappa \\
\hline Bajo & 329 & $\begin{array}{r}5,83 \\
5,25-6,50 \\
\end{array}$ & 312 & $\begin{array}{r}5,53 \\
5,0-6,16 \\
\end{array}$ & $\begin{array}{r}99,39 \\
99,16-99,62 \\
\end{array}$ & $\begin{array}{r}79,81 \\
75,19-84,42 \\
\end{array}$ & $\begin{array}{r}98,73 \\
98,40-99,05 \\
\end{array}$ & $\begin{array}{r}83,3 \\
80,00-86,0 \\
\end{array}$ \\
\hline Normal & 5.280 & $\begin{array}{r}93,63 \\
92,96-94,24 \\
\end{array}$ & 4.882 & $\begin{array}{r}86,58 \\
85,67-87,44 \\
\end{array}$ & & & & \\
\hline Macrosomia & 30 & $\begin{array}{r}0,53 \\
0,04-0,76 \\
\end{array}$ & 27 & $\begin{array}{r}0,48 \\
0,30-0,70 \\
\end{array}$ & $\begin{array}{r}99,9 \\
99,81-100,00 \\
\end{array}$ & $\begin{array}{r}77,78 \\
60,24-95,31 \\
\end{array}$ & $\begin{array}{r}99,8 \\
99,78-99,99 \\
\end{array}$ & $\begin{array}{r}79,14 \\
67,10-91,20 \\
\end{array}$ \\
\hline Missing & - & - & 418 & $\begin{array}{r}7,41 \\
6,75-8,12 \\
\end{array}$ & & & & \\
\hline Total & 5.639 & 100 & 5.639 & 100 & & & & \\
\hline \multicolumn{9}{|l|}{ PEG } \\
\hline $\mathrm{Si}$ & 56 & $\begin{array}{r}1,00 \\
0,77-1,13 \\
\end{array}$ & 99 & $\begin{array}{r}1,76 \\
1,45-2,14\end{array}$ & $\begin{array}{r}99,61 \\
99,42-99,80 \\
\end{array}$ & $\begin{array}{r}23,6 \\
14,21-32,98 \\
\end{array}$ & $\begin{array}{r}98,55 \\
98,20-98,90 \\
\end{array}$ & $\begin{array}{r}41,08 \\
31,00-51,10 \\
\end{array}$ \\
\hline No & 5.570 & $\begin{array}{r}99,00 \\
90,70-99,23 \\
\end{array}$ & 4.645 & $\begin{array}{r}81,89 \\
81,57-83,55 \\
\end{array}$ & & & & \\
\hline Missing & - & שים, & 881 & $\begin{array}{r}15,66 \\
14,73-16,63 \\
\end{array}$ & & & & \\
\hline Total & $5.626^{*}$ & 100 & 5.625 & 100 & & & & \\
\hline \multicolumn{9}{|l|}{ GEG } \\
\hline $\mathrm{Si}$ & 846 & $\begin{array}{r}15,04 \\
14,13-16,00 \\
\end{array}$ & 659 & $\begin{array}{r}11,62 \\
10,91-12,59 \\
\end{array}$ & $\begin{array}{r}95,89 \\
95,27-96,51 \\
\end{array}$ & $\begin{array}{r}81,79 \\
78,77-84,81 \\
\end{array}$ & $\begin{array}{r}97,03 \\
96,49-97,56 \\
\end{array}$ & $\begin{array}{r}75,38 \\
72,7-78,1 \\
\end{array}$ \\
\hline No & 4.780 & $\begin{array}{r}84,96 \\
84,00-85,87 \\
\end{array}$ & 4.085 & $\begin{array}{r}72,61 \\
71,44-73,77 \\
\end{array}$ & & & & \\
\hline Missing & - & - & 881 & $\begin{array}{r}15,66 \\
14,73-16,63\end{array}$ & & & & \\
\hline Total & 5.626 & 100 & 5.625 & 100 & & & & \\
\hline \multicolumn{9}{|c|}{$\begin{array}{l}{ }^{*} \text { Las diferencias de tamano muestral entre las fuentes se debe a una obsevación en la que en la fuente del INE co- } \\
\text { rresponde a la semana } 43 \text { de gestación. }{ }^{\dagger} \text { Para llevar a cabo medidas de validez, excluímos la categoría missing en } \\
\text { PEG y GEG. } \\
\text { PEG: pequeño para la edad gestacional. GEG: grande para la edad gestacional. VPP: valor predicitivo positivo }\end{array}$} \\
\hline
\end{tabular}

ción de las medidas de peso relativo (PEG y GEG). Ambos progenitores de origen extranjero $(\mathrm{OR}=1,78)$, madres sin información de empleo $(\mathrm{OR}=1,66)$ o dependientes económicamente $(\mathrm{OR}=1,47)$ y padre sin información de empleo $(\mathrm{OR}=2,33)$ son las categorías asociadas a una mayor probabilidad de no declarar la información de peso y edad gestacional. Por el contrario, las mujeres de menos de 24 años $(\mathrm{OR}=0,77)$ y las casadas $(\mathrm{OR}=0,80)$ fueron las que menor probabilidad tenían de hacerlo. Registrar el nacimiento el día después del mismo está asociado a una mayor probabilidad de aportar la información sobre el peso y la edad gestacional.
Padres sin información sobre su situación laboral $(\mathrm{OR}=1,78)$ fue la variable asociada a una mayor probabilidad de incorrecta clasificación de PEG. Por el contrario, tener al menos un progenitor de nacionalidad española $(\mathrm{OR}=0,37)$ y tratarse de una pareja casada $(\mathrm{OR}=0,58)$ se asoció a una menor probabilidad de incorrecta clasificación de PEG. Tener nacionalidad extranjera uno $(\mathrm{OR}=2,0)$ o ambos $(\mathrm{OR}=2,24)$ progenitores fue la única variable asociada a una mayor probabilidad de incorrecta declaración de GEG, mientras que las parejas casadas $(\mathrm{OR}=0,75)$ y las madres que ya tenían un niño anterior $(\mathrm{OR}=0,61)$ se asoció a una menor probabilidad de incorrecta clasificación de GEG. 


\begin{tabular}{|c|c|c|c|c|c|c|}
\hline \multicolumn{7}{|c|}{$\begin{array}{l}\text { Tabla } 2 \\
\text { Regresiones logísticas para estimar las odd ratios (OR) y sus intervalos de confianza } \\
\text { (IC95\%) de la asociación entre no declarar información de peso y edad gestacional } \\
\text { (datos missing) y la incorrecta clasificación PEG y GEG }\end{array}$} \\
\hline & \multicolumn{2}{|c|}{ Datos missing } & \multicolumn{2}{|c|}{$\begin{array}{l}\text { Clasificación incorrecta } \\
\text { de PEG }\end{array}$} & \multicolumn{2}{|c|}{$\begin{array}{l}\text { Clasificación incorrecta } \\
\text { de GEG }\end{array}$} \\
\hline & OR & IC $95 \%$ & OR & IC $95 \%$ & OR & IC 95\% \\
\hline \multicolumn{7}{|l|}{$\begin{array}{l}\text { Origen de los padres (nacionalidad) } \\
\text { Referencia ambos padres españoles }\end{array}$} \\
\hline Ambos progenitores extranjeros & $1,78^{\ddagger}$ & $1,47-2,15$ & 0,66 & $0,38-1,13$ & $2,24+$ & $1,65-3,04$ \\
\hline Al menos un progenitores español & 1,24 & $0,97-1,58$ & $0,37^{*}$ & $0,15-0,89$ & $2,02+$ & $1,39-2,93$ \\
\hline \multicolumn{7}{|l|}{\begin{tabular}{|l|}
$\begin{array}{l}\text { Edad de la madre (años) } \\
\text { referencia } 25-30\end{array}$ \\
\end{tabular}} \\
\hline$<24$ & $0,77^{*}$ & 0,61-0,98 & 1,41 & $0,69-2,88$ & 0,95 & $0,63-1,44$ \\
\hline $31-35$ & 1,03 & $0,84,1,27$ & 0,72 & $0,38-1,34$ & 1,15 & $0,82-1,60$ \\
\hline$>36$ & 1,10 & & 0,83 & $0,40-1,74$ & 1,13 & $0,76-1,69$ \\
\hline \multicolumn{7}{|l|}{$\begin{array}{l}\text { Edad del padre (años) } \\
\text { Referencia } 25-30\end{array}$} \\
\hline$<24$ & 1,12 & & 0,91 & $0,37-2,21$ & 0,76 & $0,45-1,27$ \\
\hline $31-35$ & 1,07 & & 0,89 & $0,47-1,70$ & 0,95 & $0,67-1,34$ \\
\hline$>36$ & 1,18 & & 1,25 & $0,63-2,50$ & 1,08 & $0,75-1,57$ \\
\hline \multicolumn{7}{|l|}{$\begin{array}{l}\text { Orden de nacimiento } \\
\text { Referencia primero }\end{array}$} \\
\hline Distinto de primero & 1,08 & & 0,93 & $0,58-1,48$ & $0,61^{\ddagger}$ & $0,47-0,80$ \\
\hline \multicolumn{7}{|l|}{$\begin{array}{l}\text { Sexo del nacido } \\
\text { Referencia varones }\end{array}$} \\
\hline Mujeres & 0,98 & & 1,32 & $0,85-2,03$ & 0,88 & $0,69-1,12$ \\
\hline \multicolumn{7}{|l|}{$\begin{array}{l}\text { PEG } \\
\text { Referencia no }\end{array}$} \\
\hline $\mathrm{Si}$ & 0,78 & & & & & \\
\hline \multicolumn{7}{|l|}{$\begin{array}{l}\text { GEG } \\
\text { Referencia no }\end{array}$} \\
\hline Sí & 0,91 & & & & & \\
\hline \multicolumn{7}{|l|}{$\begin{array}{l}\text { Ocupación de la madre } \\
\text { Referencia no trabajadora }\end{array}$} \\
\hline Dependiente económicamente & $1,47^{\ddagger}$ & $1,17-1,85$ & 1,28 & $0,70-2,36$ & 1,08 & $0,77-1,51$ \\
\hline Missing & $1,66^{\ddagger}$ & $1,36-2,04$ & 0,96 & $0,52-1,80$ & 0,88 & $0,62-1,23$ \\
\hline \multicolumn{7}{|l|}{$\begin{array}{l}\text { Ocupación del padre } \\
\text { Referencia no trabajador }\end{array}$} \\
\hline Dependiente económicamente & 1,58 & \begin{tabular}{|l|}
$0,69-3,62$ \\
\end{tabular} & 2,44 & $0,31-19,0$ & 1,04 & $0,24-4,44$ \\
\hline Missing & $2,33^{\ddagger}$ & $1,94-2,80$ & $1,78^{*}$ & $1,01-3,12$ & 1,06 & $0,77-1,46$ \\
\hline \multicolumn{7}{|l|}{$\begin{array}{l}\text { Estado civil de la madre } \\
\text { Referencia no casada }\end{array}$} \\
\hline Casada & $0,80 *$ & & $0,58^{*}$ & $0,34,0,99$ & $0,75^{*}$ & $0,57-0,99$ \\
\hline \multicolumn{7}{|c|}{$\begin{array}{l}\text { Días desde el nacimiento al registro del nacimiento } \\
\text { Referencia no mismo día }\end{array}$} \\
\hline 2-4 días & $0,40^{\ddagger}$ & & 0,97 & $0,34-2,81$ & 1,30 & $0,64-2,64$ \\
\hline 5-10 días & $0,37 \div$ & & 0,91 & $0,31-2,65$ & 1,15 & $0,56-2,34$ \\
\hline más de 10 días & $0,44 \div$ & & 0,66 & $0,20-2,14$ & 1,15 & $0,55-2,42$ \\
\hline Número de observaciones & 5.625 & & 4.744 & & 4.744 & \\
\hline Prob $>$ chi $^{2}$ & 0,0000 & & 0,1338 & & 0,0000 & \\
\hline Pseudo $\mathrm{R}^{2}$ & 0,0761 & & 0,0287 & & 0,0291 & \\
\hline
\end{tabular}




\section{DISCUSIÓN}

En relación a las medidas de salud perinatal, los resultados de este estudio muestran que los datos del INE son más fiables cuando se utilizan medidas de peso absoluto (bajo peso, macrosomia). Los indicadores de peso relativo, especialmente PEG, se ven afectados en mayor medida por la proporción de datos faltantes (principalmente por omisión de la edad gestacional) y por la incorrecta declaración de la información. Los datos del INE tienden a sobreestimar la proporción de PEG y a infraestimar la proporción de GEG. Estos resultados desaconsejan la utilización de esta fuente para construir curvas de crecimiento representativas a nivel nacional.

La omisión de las variables peso y edad gestacional no es aleatoria sino que está asociada a la nacionalidad y a la situación laboral de los progenitores. Este resultado pone en evidencia un sesgo de selección de la información en función de variables sociodemográficas. Esto quiere decir que la salud perinatal valorada con los datos del INE no representa igualmente a todos los colectivos. De esto se infiere que los estudios orientados a evaluar las desigualdades sociales en PEG y GEG tienen que ser interpretados con cautela. El hecho de que la ausencia de información sobre peso al nacer y edad gestacional sea casi dos veces más probable entre progenitores extranjeros pone de manifiesto que los procedimientos para la recogida de la información podrían no estar considerando las necesidades de la población inmigrante. Este resultado insinúa que podrían existir barreras que deberían conocerse y eliminarse (por ejemplo, idiomáticas, de conocimiento del procedimiento).

Nuestros resultados sugieren que la incorrecta clasificación de PEG y GEG se debe a errores sistemáticos, al ocurrir con mayor probabilidad entre los padres que no declaran su empleo o que están en situación de dependencia y entre las mujeres que no están casadas (con respecto a PEG) y fundamentalmente entre los progenitores de nacionalidad extranjera (en relación a GEG). Este resultado alerta sobre la necesidad de estudiar las causas por las que estos colectivos declararían incorrectamente la información del peso al nacer y la edad gestacional, dando lugar a una incorrecta clasificación de las medidas estudiadas (PEG, GEG) lo que podría sugerir, por ejemplo, un menor seguimiento prenatal entre los grupos más vulnerables (inmigrantes, mujeres solteras y progenitores sin empleo).

La principal limitación de este estudio es que sólo cuenta con información relativa a un hospital de Madrid y, por lo tanto, los resultados no pueden ser directamente extrapolables ni al total de la comunidad autónoma ni al conjunto de España. En este sentido, se necesitan mayores esfuerzos para conseguir una validación nacional que evalué además las diferencias regionales. Otra limitación es que la información estudiada se extrajo del Boletín de Partos vigente durante el periodo 1995-2006 (el nuevo boletín se introdujo en el año 2007) 9 . Sin embargo, aunque el nuevo Boletín recoge información adicional (por ejmplo educación y país de nacimiento de los progenitores) ni los procedimientos ni la recogida de la información ni la formulación de las preguntas de nuestro interés han sufrido modificaciones mayores. En este sentido, creemos que las recomendaciones derivadas de este estudio podrían seguir siendo de actualidad.

A pesar de estas limitaciones, nuestro estudio tiene importantes fortalezas. Por un lado, se realizó con la única base de datos existente hasta la fecha, la cual cuenta con la información cruzada a nivel individual entre el INE y un hospital, garantizando la comparación de la información. Asimismo, aunque se han realizado importantes esfuerzos para evaluar la calidad de la información del INE en materia de salud perinatal ${ }^{8,16}$ y se ha llamado la atención sobre la importancia de las referencias que se utilizan en la estimación de $\mathrm{PEG}^{17}$, este es el primer estudio que discute la elección de indicadores relativos y absolutos de peso a la luz de la calidad de los datos orientado a mejorar la investigación epidemiológica. 
En conclusión, la calidad de la información disponible debería ser considerada a la hora de elegir el indicador más adecuado para valorar la salud perinatal. De este modo, aunque PEG es teóricamente el mejor indicador de crecimiento retardado intrauterino no resulta ser el más indicado cuando la calidad de los datos no está garantizada. Las conclusiones de este trabajo aconsejan mejorar las estadísticas de nacimientos y, para ello, proponen que en el futuro la información sobre el peso y la edad gestacional se recoja directamente de fuentes hospitalarias.

\section{BIBLIOGRAFÍA}

1. Wilcox AJ. On the Importance -and the unimportance- of Birthweight. Int J Epidemiol. 2001;30:1233-41.

2. Lubchenco LO, Hansman C, Dresserm M, Boyd E. Growth as estimated from liveborn birth-weight data at 24 to 42 weeks gestation. Pediatrics. 1963;32:763-800.

3. Urquia ML, Ray JG. Seven caveats on the use of low birthweight and related indicators in health research. J Epidemiol Community Health. 2012;66(11):971-5.

4. Weiner Z, Ben-Shlomo I, Beck-Fruchter R, Goldberg Y, Shalev E. Clinical and ultrasonographic weight estimation in large for gestational age fetus. Eur J Obstet Gynecol Reprod Biol. 2002;105(1):20-24.

5. Walsh JM, McAuliffe FM. Prediction and prevention of the macrosomic fetus. Eur J Obstet Gynecol Reprod Biol. 2012;162(2):125-30.

6. Urquia ML, Frank JW, Alazragui M, Guevel C, Spinelli $\mathrm{H}$. Contrasting socioeconomic gradients in small for gestational age and preterm birth in Argentina, 2003-2007. Int. J. Public Health. 2013;58(4):529-36.

7. Juárez S. Notas acerca del movimiento natural de la población para el estudio de la salud perinatal. Gac Sanit. 2014;28(6):505-7.

8. Juárez S, Alonso Ortíz T, Ramiro Fariñas D, Bolúmar F. The quality of vital statistics for studying perinatal health: the Spanish case. Paediatr Perinat Epidemiol. 2012;26(4):310-15.

9. INE. Estadisticas del Movimiento Natural de la Población. Metodología. Madrid: Instituto Nacional de Estadística; 2013.

10. Sesma E, Sánchez A, Núñez D. El estudio longitudinal de extranjeros de la Comunidad de Madrid. Jornadas de Estadística de las Comunidades Autónomas, JECAS. Santander: JECAS; 2008.
11. Bohensky M, A, Jolley D, Sundararajan V, Evans $\mathrm{S}$, Ibrahim J, Brand C. Development and validation of reporting guidelines for studies involving data linkage. Aust NZJ Public Health. 2011;35(5):486-9.

12. Carrascosa Lezcano A, Ferrández Longás A, Yeste Fernández D, García- Dihinx Villanova J, Romo Montejo A, Copil Copil A, et al. Estudio transversal español de crecimiento 2008. Parte I: valores del peso y longitud en recién nacidos de 26-42 semanas de edad gestacional. An Pediatr (Barc). 2008;68(6):544-51.

13. Carrascosa Lezcano A, Fernández JM, Fernández A, López-Siguero JP, Sánchez E, Sobradillo B, et al. Estudio transversal español de crecimiento 2008. Parte II: valores del peso y longitud en recién nacidos de 26-42 semanas de edad gestacional. An Pediatr (Barc).2008;68(6):552-69.

14. Dollberg S, Haklai Z, Minmouni B, Gorfei I, Gordon E-S. Birth weight standards in the live-born population in Israel. IMAJ. 2005;7:311-14.

15. Ticona Rendón M, Huanco Apaza D. Curvas de crecimiento intrauterino propias del peru y su efecto en la identificación de una nueva población neonatal de alto riesgo nutricional. Lima: Ministerio de Salud; 2007.

16. Río Sánchez I, Castelló A, Jané M, Prats R, Barona $\mathrm{C}$, Más $\mathrm{R}$, et al. Calidad de los datos utilizados para el cálculo de indicadores de salud reproductiva y perinatal en población autóctona e inmigrante. Gac Sanit. 2010;24(2):172-7

17. Ayerza Casas A, Rodríguez Martínez G, Samper Villagrasa MP, Ventura Faci P. Nacer pequeño para la edad gestacional puede depender de la curva de crecimiento utilizada. Nutr Hosp. 2011;26(4):752-8. 